\title{
Shadow of a Kerr-like black hole
}

\author{
Farruh Atamurotov \\ Institute of Nuclear Physics, Ulughbek, Tashkent 100214, Uzbekistan \\ email: farruh@astrin.uz
}

\begin{abstract}
The shadow of a Kerr-like black hole has been considered and it was shown that in addition to the specific angular momentum $a$, deformation parameter of Kerr-like space-time essentially deforms the shape of the black hole shadow. For a given value of the black hole spin parameter $a$, the presence of a deformation parameter $\epsilon$ reduces the shadow and enlarges its deformation with respect to the one in the Kerr space-time.
\end{abstract}

Keywords. Kerr-like space-time, Photon motion, Shadow of black hole.

\section{Introduction}

Recently, such a model was suggested by Johannsen \& Psalti (2009) who considered deviations from the Schwarzschild and Kerr solutions and found a regular outside the event horizon space-time, which reduces to the Kerr one, when the deformation parameters vanish. The Johannsen and Psalti's metric is not a vacuum solution of the Einstein equations, but is obtained in a kind of perturbative way in order to include various possible deviations from the Kerr solution in alternative theories of gravity.

A black hole is not visible, it may be observable nonetheless since it may create a shadow if it is in front of a bright background. The apparent shape of an extremely rotating black hole has been first studied by Bardeen (1973). Recently, shadow of black hole was investigated by several authors (e.g. Atamurotov et al. 2013).

\section{Photon motion around Kerr-like black hole}

The deformed Kerr-like metric which describes a stationary axisymmetric, and asymptotically flat vacuum space-time, in the standard Boyer-Lindquist coordinates, can be expressed as Johannsen \& Psalti (2009)

$$
\begin{aligned}
\mathrm{d} s^{2}= & -\left(1-\frac{2 M r}{\Sigma^{2}}\right)(1+h) \mathrm{d} t^{2}+\frac{\Sigma^{2}(1+h)}{\Delta+a^{2} h \sin ^{2} \theta} \mathrm{d} r^{2}+ \\
& \Sigma^{2} \mathrm{~d} \theta^{2}-\frac{4 a M r \sin ^{2} \theta}{\Sigma^{2}}(1+h) \mathrm{d} t \mathrm{~d} \phi+ \\
& \sin ^{2} \theta\left[\Sigma^{2}+\frac{a^{2}\left(\Sigma^{2}+2 M r\right) \sin ^{2} \theta}{\Sigma^{2}}(1+h)\right] \mathrm{d} \phi^{2},
\end{aligned}
$$

where

$$
\begin{aligned}
\Sigma^{2} & =r^{2}+a^{2} \cos ^{2} \theta, \\
\Delta & =r^{2}-2 M r+a^{2} . \\
h & =\frac{\epsilon M^{3} r}{\Sigma^{4}},
\end{aligned}
$$



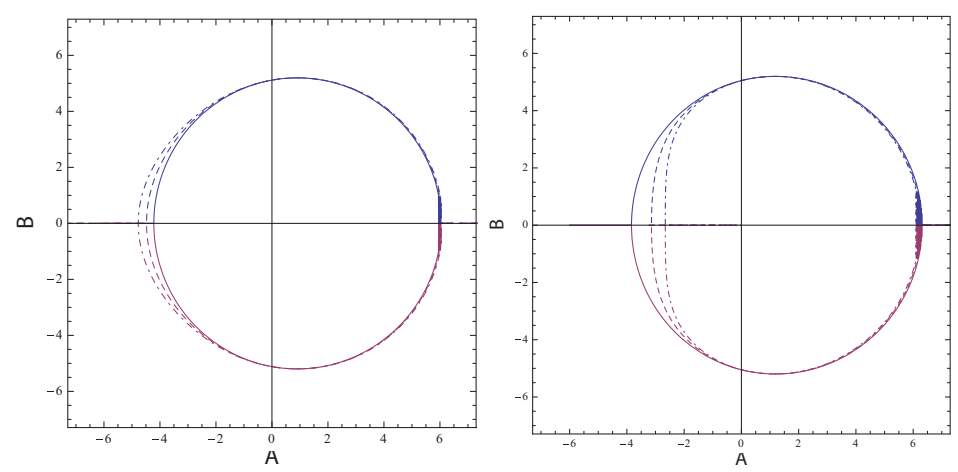

Figure 1. Shadow cast by a Kerr-like black hole situated at the origin of coordinates with inclination angle $\theta=\pi / 2$, having a rotation parameter $a$ and a deformation parameter $\epsilon$. Left panel: for $a / M=0.45, \epsilon=0$ (solid line), $\epsilon=-5$ (dashed line), $\epsilon=-10$ and right panel: $a / M=0.6$ and $\epsilon=0$ (solid line), $\epsilon=5$ (dashed line), $\epsilon=10$ (dashed-dotted line).

Using the celestial coordinates one can easily describe the shadow (see for example Vázquez \& Esteban 2004):

$$
\alpha=\lim _{r_{0} \rightarrow \infty}\left(-r_{0}^{2} \sin \theta_{0} \frac{\mathrm{d} \phi}{\mathrm{d} r}\right)
$$

and

$$
\beta=\lim _{r_{0} \rightarrow \infty} r_{0}^{2} \frac{\mathrm{d} \theta}{\mathrm{d} r}
$$

In Fig. 1, we show the contour of the shadows of black holes with rotation parameters and for several values of the deformation parameter $\epsilon$.

\section{Conclusion}

We have analyzed how the shadow of the black hole is distorted by the presence of the deformation parameter $\epsilon$ (Atamurotov et al. 2013). We have shown that with increasing deformation parameter (Atamurotov et al. 2013), the radius of the shadow of the black hole decreases. This phenomena also related to the fact that the increase of deformation parameter forces photon orbits to come closer which corresponds to the decrease of gravitational force acting on photons. In the case of negative $\epsilon$ the deflected photons with particular value of impact parameter could be absorbed by central object while they could escape in pure Kerr black hole case with the same impact parameter. The increase of distortion parameter $\delta_{s}$ with the increase of module of deformation parameter $\epsilon$ corresponds to deviation of the shape of shadows from pure circle (Atamurotov et al. 2013). The deformed rotating black hole's shadow is also going to be deformed independently on sign of deformation parameter.

\section{References}

Atamurotov, F., Abdujabbarov, A., \& Ahmedov, B. 2013, Phys. Rev. D, 88, 064004.

Bardeen, J. M., Black holes: École D'été de Physique Théorique, Les Houches 1972, 215-239, Gordon and Breach Science Publishers, New York, 1973.

Johannsen, T. \& Psaltis, D. 2011, Phys. Rev. D, 83, 124015.

Vázquez S. \& Esteban, E. 2004, Nuovo Cim. 119B, 489. 